\title{
Lattice mode symmetry analysis of the orthorhombic phase of methylammonium lead iodide using polarized Raman
}

\author{
Rituraj Sharma ${ }^{1},{ }^{1, *}$ Matan Menahem $\odot,{ }^{1,}{ }^{*}$ Zhenbang Dai $\odot,{ }^{2}$ Lingyuan Gao, ${ }^{2}$ Thomas M. Brenner $\odot,{ }^{1}$ Lena Yadgarov $\odot,{ }^{1}$ \\ Jiahao Zhang, ${ }^{2}$ Yevgeny Rakita, ${ }^{1}$ Roman Korobko $\odot,{ }^{1}$ Iddo Pinkas $\odot,{ }^{1}$ Andrew M. Rappe ${ }^{2, \dagger}$ and Omer Yaffe $\odot^{1, \ddagger}$ \\ ${ }^{1}$ Department of Materials and Interfaces, Weizmann Institute of Science, Rehovot 7610001, Israel \\ ${ }^{2}$ Department of Chemistry, University of Pennsylvania, Philadelphia, Pennsylvania 19104, USA
}

(Received 6 December 2019; revised manuscript received 18 February 2020; accepted 4 March 2020; published 4 May 2020)

\begin{abstract}
In the last decade, hybrid organic-inorganic halide perovskites have emerged as a new type of semiconductor for photovoltaics and other optoelectronic applications. Unlike standard, tetrahedrally bonded semiconductors (e.g., Si and GaAs), the ionic thermal fluctuations in the halide perovskites (i.e., structural dynamics) are strongly coupled to the electronic dynamics. Therefore, it is crucial to obtain accurate and detailed knowledge about the nature of the atomic motions within the crystal. This has proved to be challenging due to low thermal stability and the complex, temperature-dependent structural phase sequence of the halide perovskites. Here, these challenges are overcome and a detailed analysis of the low-frequency lattice mode symmetries is provided in the low-temperature orthorhombic phase of methylammonium-lead iodide. Raman measurements using linearly and circularly polarized light at $1.16 \mathrm{eV}$ excitation are combined with density functional perturbation theory (DFPT). By performing an iterative analysis of Raman polarization-orientation dependence and DFPT mode analysis, the crystal orientation is determined. Subsequently, accounting for birefringence effects detected using circularly polarized light excitation, the symmetries of all of the observed Raman-active modes at $10 \mathrm{~K}$ are assigned.
\end{abstract}

DOI: 10.1103/PhysRevMaterials.4.051601

Methylammonium lead iodide (MAPI) has emerged as an attractive material for next-generation photovoltaic technologies, owing to its optimal band gap and excellent photoconversion efficiency [1-5]. From a fundamental standpoint, MAPI and other hybrid organic-inorganic halide perovskites (HOPs) are intriguing because unlike standard semiconductors, the optical and charge transport properties of HOPs are considered to be strongly coupled to their lattice fluctuations [6-10].

Indeed, there has been intensive research dedicated to deciphering the structural characteristics of halide perovskites, employing x-ray diffraction (XRD), Raman and neutron scattering, dielectric measurements, etc. [11-15]. Specifically, Raman scattering is a highly adaptable technique that gives qualitative and quantitative insights into local structure and dynamics. The Raman spectrum of a hybrid (i.e., organicinorganic) material can be divided into two regimes. The first is high-frequency, molecular (internal) vibrations and the second is the low-frequency lattice (external) vibrations [16]. Only the latter carries information about the lattice modes $[17,18]$.

\footnotetext{
${ }^{*}$ These authors contributed equally to this work.

${ }^{\dagger}$ rappe@sas.upenn.edu

‡omer.yaffe@weizmann.ac.il
}

Published by the American Physical Society under the terms of the Creative Commons Attribution 4.0 International license. Further distribution of this work must maintain attribution to the author(s) and the published article's title, journal citation, and DOI.
There are many recent reports discussing the Raman spectra of HOPs [7,19-21]. Density functional theory (DFT) and molecular dynamics (MD) based calculations have been used to assign the Raman-active modes [20,22,23]. However, Raman measurements on MAPI have proven to be very challenging both from the experimental and computational standpoints. Regarding the experiment, the high light absorption coefficient and low thermal stability of MAPI do not allow long exposure to intense visible light without inducing irreversible sample damage [24-26]. This is true even for near-IR, $1.58 \mathrm{eV}$ light [27]. Furthermore, the complex crystal structure and temperature phase sequence do not permit an unambiguous determination of the crystallographic orientation at low temperatures without a separate XRD measurement in identical conditions. Regarding DFPT computation, the complex crystal structure and the large number of atoms in the unit cell result in a very large number of Raman-active modes that are closely spaced in energy. Therefore, it is very difficult to determine by DFPT which specific modes are actually observed in experiment without prior knowledge of the mode symmetries.

Here, we overcome the aforementioned challenges by performing an iterative analysis between polarization-orientation (PO) Raman scattering measurements with $1.16 \mathrm{eV}$ laser excitation and DFPT computation. We provide an unambiguous determination of the Raman tensors for all the observed Raman-active, low frequency lattice modes as well as the crystallographic orientation in MAPI at $10 \mathrm{~K}$. Furthermore, by combining our PO measurements with circularly polarized light excitation, we are able to account for the effect of 


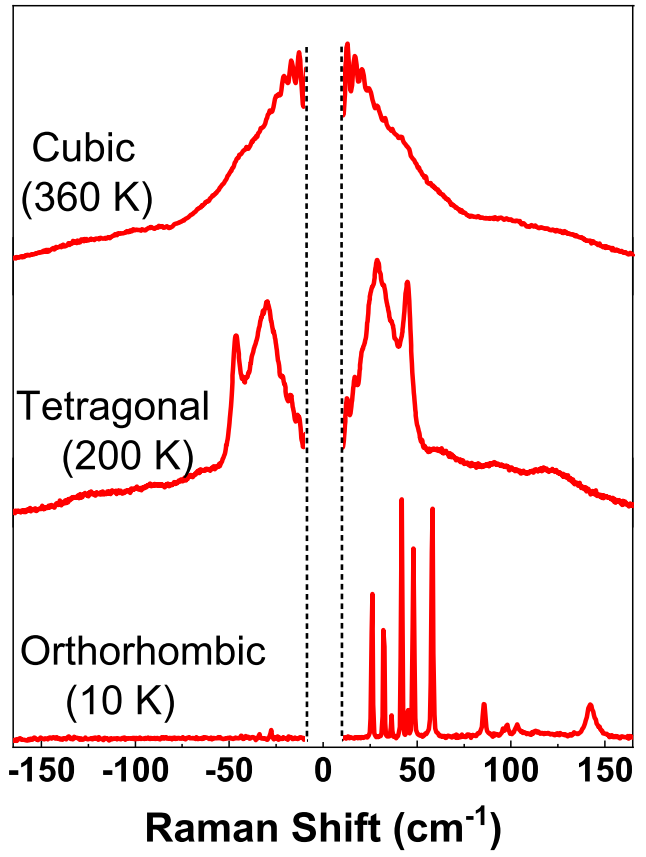

FIG. 1. Temperature dependence of low-frequency unpolarized Raman spectra showing the phase transitions in MAPI. The Rayleigh scattering region of the spectra is removed from $0 \mathrm{~cm}^{-1}$ to the vertical dotted lines.

birefringence on the Raman signal. Thus, we demonstrate the great potential of our measurements and analysis as an exceptional tool for vibrational mode assignments in highly complicated systems like HOPs.

MAPI has an $A B X_{3}$ perovskite structure, with $\mathrm{PbI}_{6}$ in corner-sharing octahedra and $\mathrm{MA}^{+}$inside the cuboctahedral cavities. It is known to have three structural phases: an orthorhombic (Pnma) phase below $162 \mathrm{~K}$, a tetragonal $(I 4 / \mathrm{mcm})$ phase between 162 and $327 \mathrm{~K}$, and a cubic $(P m-3 m)$ structure beyond $327 \mathrm{~K}[28,29]$.

Figure 1 shows the low-frequency unpolarized Raman spectra of MAPI single crystals taken across the phase sequence covering simultaneously both Stokes and anti-Stokes scattering. The spectral features (molecular modes) beyond $\pm 200 \mathrm{~cm}^{-1}$ are beyond the scope of the present work [16]. The spectrum of the orthorhombic phase $(10 \mathrm{~K})$ shows sharp peaks, and the orthorhombic-tetragonal phase transition is characterized by an abrupt change, where the sharp peaks are replaced by relatively broad features.

The Raman spectra in the tetragonal and cubic phases are envelopes of the orthorhombic phase spectrum. Therefore, to interpret the nature of atomic motions in the high-temperature phases, a thorough understanding of the vibrational characteristics of the low-temperature phase is a prerequisite.

\section{PO RAMAN SCATTERING ANALYSIS}

The atomic vibrations in a crystal are closely related to the crystallographic arrangement of atoms and have specific symmetries. Group theory provides tools to predict the symmetries of the vibrational normal modes by decomposing the crystal space group to its irreducible representations. According to factor group analysis, we expect 18 Raman-active lattice modes in the orthorhombic MAPI structure with the following irreducible representation [30,31]:

$$
\Gamma=5 A_{g}+4 B_{1 g}+5 B_{2 g}+4 B_{3 g}
$$

The Raman tensor of each mode, which relates the scattering cross section to light polarization vector, must obey the symmetry of the irreducible representation as well $[18,32]$. The corresponding Raman tensors are given as

$$
\begin{aligned}
A_{g} & =\left(\begin{array}{lll}
a & 0 & 0 \\
0 & b & 0 \\
0 & 0 & c
\end{array}\right), \quad B_{1 g}=\left(\begin{array}{lll}
0 & d & 0 \\
d & 0 & 0 \\
0 & 0 & 0
\end{array}\right), \\
B_{2 g} & =\left(\begin{array}{lll}
0 & 0 & e \\
0 & 0 & 0 \\
e & 0 & 0
\end{array}\right) \text { and } B_{3 g}=\left(\begin{array}{lll}
0 & 0 & 0 \\
0 & 0 & f \\
0 & f & 0
\end{array}\right)
\end{aligned}
$$

To extract the Raman tensor for each mode, we perform PO Raman measurements. Briefly, the crystal is excited by planepolarized laser light having polarization $e_{i}$. The scattered light $e_{s}$ is then filtered by another polarizer (analyzer) for polarizations parallel and perpendicular to the incident light. This measurement is repeated after rotating the polarization of the incident light. The Raman tensor for each peak can be identified from the angular dependence of the peak intensity according to [33]:

$$
I(\theta) \propto\left|e_{s}(\theta) \mathcal{R}^{T} R \mathcal{R} e_{i}(\theta)\right|^{2}
$$

where $e_{i}$ and $e_{s}$ are the light polarization vectors which are either parallel or perpendicular to one another, and $\mathcal{R}$ is a rotation matrix transforming the Raman tensor $R$ from the crystal system to the laboratory system.

Figure 2(a) shows the contour plot of the low-frequency Raman spectra of MAPI acquired at different excitation polarization angles. $\theta=0^{\circ}$ is an arbitrary angle with respect to the sample axes, determined by the experimental setup. The upper panel shows the averaged Raman spectrum (over all angles), while the middle and lower panels show the spectra in parallel and cross configurations, respectively. A periodic modulation in intensity as a function of polarization angle is apparent for all Raman-active modes. All the modes in parallel configuration show a periodicity of $180^{\circ}$ except the mode at $\approx 98 \mathrm{~cm}^{-1}$, which shows a $90^{\circ}$ periodicity. In the cross configuration, all modes possess a $90^{\circ}$ periodicity. It is worth mentioning that all the data presented here are in raw form without any normalization or base-line correction. The absence of any artifacts, intensity leakage, or drift demonstrates the excellent stability of our Raman setup and the absence of material evolution or damage during the experiment. Each Raman spectrum was deconvolved using a multi-Lorentz oscillator model (details in Supplemental Material (SM) [34]) and the intensity angular dependence was extracted.

Importantly, to exclude the possibility of scattering by surface defects and domain boundaries, we performed the same PO experiment on several MAPI crystals at different locations (Fig. S6), showing the same polarization dependence. This demonstrates that the observed dependence is not a result of randomly distributed surface defects or domain boundaries.

The quantitative analysis of the angular dependence of the scattered intensity using Eq. (2) requires prior knowledge 
(a)
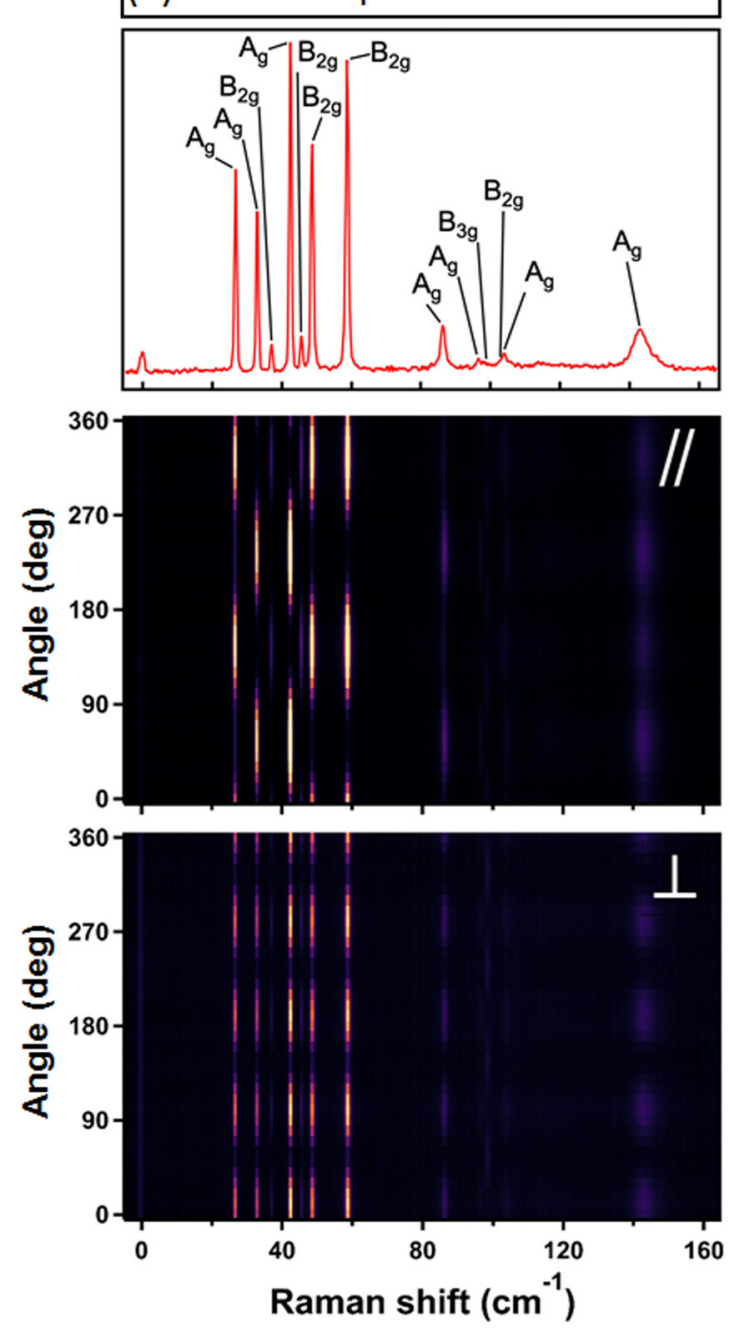
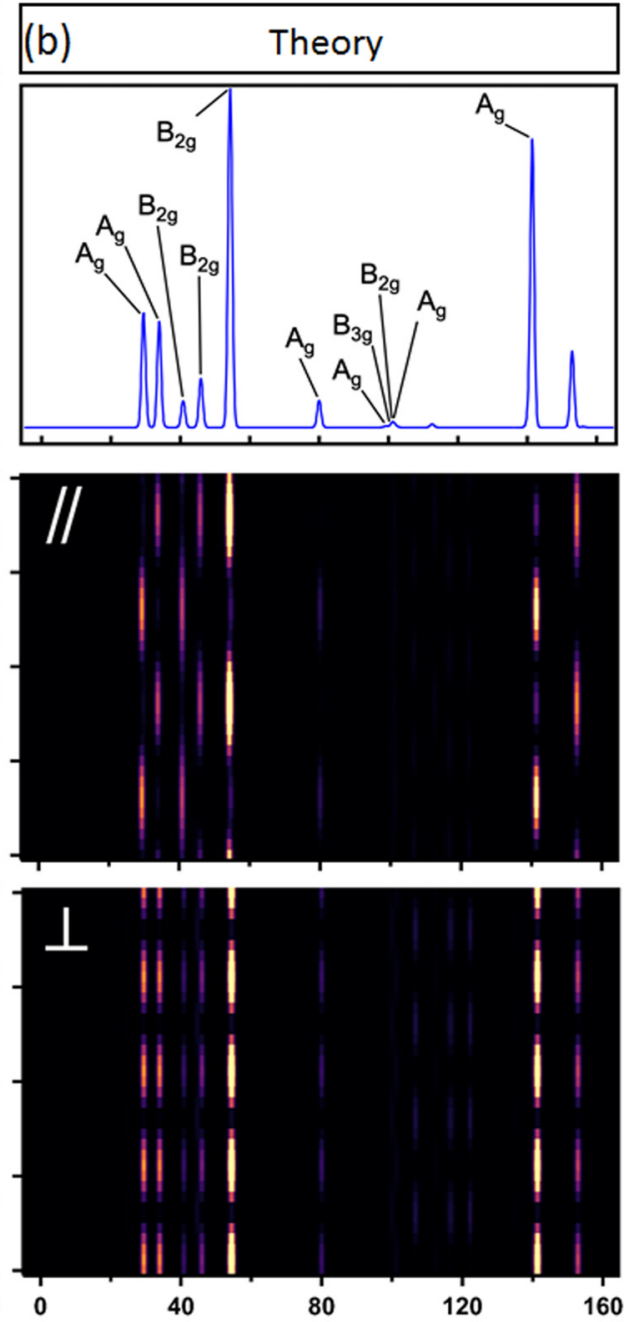

Raman shift $\left(\mathrm{cm}^{-1}\right)$

FIG. 2. (a) Experimental and (b) theoretically calculated angular dependence of low-frequency polarized Raman spectra of the orthorhombic phase of MAPI at $10 \mathrm{~K}$. The top panel shows the averaged spectra (over all angles), middle panel shows parallel and lower panel shows cross configuration for experiment and theory, respectively. $\theta=0^{\circ}$ is an arbitrary angle and theory is rotated to align with experiment.

of the crystal face probed in the PO Raman experiment. As MAPI changes phase to a lower symmetry (spontaneous symmetry breaking) as it is cooled, XRD taken at room temperature doesn't provide the proper orientation of the crystal surface. Therefore, it is necessary to obtain exact information about the crystal orientation at cryogenic temperature using XRD at the same spot where Raman is probed. This is experimentally challenging. Therefore, we turned to DFPT calculations to determine the orientation of the crystal surface.

\section{DETERMINATION OF ILLUMINATED FACE AND MODE ASSIGNMENTS USING DFPT}

To determine the orientation of the illuminated face, we first used $a b$ initio DFPT to calculate the unpolarized Raman spectrum in the low-frequency regime as shown in the top panel of Fig. 2(b). Thereafter, the corresponding Raman tensors were derived through a procedure described in the SM [34]. Using the calculated Raman tensors, the angular dependence of the Raman intensities was simulated for different crystal orientations (see SM [34]). The experimental angular dependence best matched with that calculated along the [101] crystal orientation, as shown in Fig. 2(b) middle and lower panels. Once we determined the correct orientation, we proceeded to complete the identification of the mode symmetries and their dominant motions (Table I) by matching calculated and experimental mode frequencies and polarization dependence. The excellent agreement between experiment and DFPT calculations (Fig. 2) confirms that all the observed peaks arise from lattice modes rather than bulk defects.

Having determined the correct Raman tensor for each mode, we noticed that the angular dependencies of the $\mathrm{A}_{g}$ modes could not be satisfactorily fit with Eq. (2), although the $\mathrm{B}_{2 g}$ modes fit quite well. This is illustrated in Fig. 3(a) where the experimental data are shown by dots and the best fit to Eq. (2) is shown by dashed lines. Particularly, the secondary maximum of the $\mathrm{A}_{g}$ mode for the parallel polarization configuration was never reproduced by the fitting curves [labeled with arrow in Fig. 3(a)]. We therefore conclude that the conventional Eq. (2) fails to explain the behavior of the 
TABLE I. Frequency, symmetry, and corresponding dominant atomic motions in MAPI.

\begin{tabular}{|c|c|c|c|c|}
\hline \multirow[b]{2}{*}{ Mode } & \multicolumn{2}{|c|}{ Frequency $\left(\mathrm{cm}^{-1}\right)$} & \multirow[b]{2}{*}{ Symmetry } & \multirow[b]{2}{*}{ Dominant Motion } \\
\hline & Experiment & Theory & & \\
\hline 1 & 26.9 & 29.4 & $\mathrm{~A}_{g}$ & $\mathrm{PbI}_{3}$ Network \\
\hline 2 & 33.1 & 33.9 & $\mathrm{~A}_{g}$ & $\mathrm{PbI}_{3}$ Network \\
\hline 3 & 37.2 & 35.6 & $\mathrm{~B}_{2 g}$ & $\mathrm{PbI}_{3}$ Network \\
\hline 4 & 42.6 & 40.8 & $\mathrm{~A}_{g}$ & $\mathrm{PbI}_{3}$ Network \\
\hline 5 & 45.8 & & $\mathrm{~B}_{2 g}$ & \\
\hline 6 & 48.8 & 45.9 & $\mathrm{~B}_{2 g}$ & $\mathrm{PbI}_{3}$ Network \\
\hline 7 & 58.9 & 54.2 & $\mathrm{~B}_{2 g}$ & $\mathrm{PbI}_{3}$ Network \\
\hline 8 & 86.5 & 80.0 & $\mathrm{~A}_{g}$ & MA motion \\
\hline 9 & 96.7 & 99.3 & $\mathrm{~A}_{g}$ & $\mathrm{PbI}_{3}$ Network \\
\hline 10 & 98.7 & 100.0 & $\mathrm{~B}_{3 g}$ & $\mathrm{PbI}_{3}$ Network \\
\hline 11 & 102.7 & 101.1 & $\mathrm{~B}_{2 g}$ & $\mathrm{PbI}_{3}$ Network \\
\hline 12 & 104.4 & 101.2 & $\mathrm{~A}_{g}$ & $\mathrm{PbI}_{3}$ Network \\
\hline 13 & 143.3 & 141.4 & $\mathrm{~A}_{g}$ & MA motion \\
\hline
\end{tabular}

$\mathrm{A}_{g}$ Raman modes. To resolve this discrepancy, we now turn to circularly polarized Raman measurements.

\section{DETECTION OF BIREFRINGENCE EFFECTS USING CIRCULARLY POLARIZED RAMAN SCATTERING}

Figure 3(b) shows the Raman spectra acquired using circularly polarized light (CPL) excitation. In isotropic materials, a Raman mode would retain its light polarization even when excited with CPL (see SM for silicon as example system). The scattered CPL can be analyzed for the component rotating

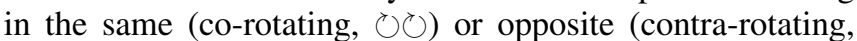
$\circlearrowright \circlearrowleft)$ direction with respect to the incident polarization. The reversal coefficient $\varrho$, along the [101] direction, is defined as [35]

$$
\varrho=\frac{I_{\circlearrowright \circlearrowleft}}{I_{\circlearrowright \circlearrowright}}=\frac{|(a+c)+2 b-2 e|^{2}}{|(a+c)-2 b-2 e-i \sqrt{8}(d-f)|^{2}}
$$

where $I_{\circlearrowright \circlearrowleft}$ and $I_{\circlearrowright \circlearrowright}$ are the scattering intensities of the counter-rotating and co-rotating components, respectively, and $a, b, c, d, e, f$ are the components of the Raman tensors defined in Sec. I. It is apparent that $\varrho=1$ for all observed modes, except the $98.7 \mathrm{~cm}^{-1}$ mode which presents $\varrho=0$. The reversal coefficient 1 indicates that the polarization of the CPL is not maintained after scattering. We hypothesize that this is a manifestation of the birefringence effect arising from the anisotropic properties of HOPs [36-39]. To test our hypothesis, we modify Eq. (2) to accommodate birefringence effects $[33,40,41]$ :

$$
I(\theta) \propto\left|e_{s}(\theta+\delta) J \mathcal{R}^{T} R \mathcal{R} J e_{i}(\theta+\delta)\right|^{2}
$$

and

$$
J=\left(\begin{array}{ccc}
1 & 0 & 0 \\
0 & e^{i\left|\phi_{y}\right|} & 0 \\
0 & 0 & e^{i\left|\phi_{z}\right|}
\end{array}\right)
$$

where $J$ is the Jones matrix of light propagation in the material [42]. $\left|\phi_{y}\right|=\left|\phi_{y y}-\phi_{x x}\right|$ and $\left|\phi_{z}\right|=\left|\phi_{z z}-\phi_{x x}\right|$ are the relative phases that the electric field components accumulate during (a)
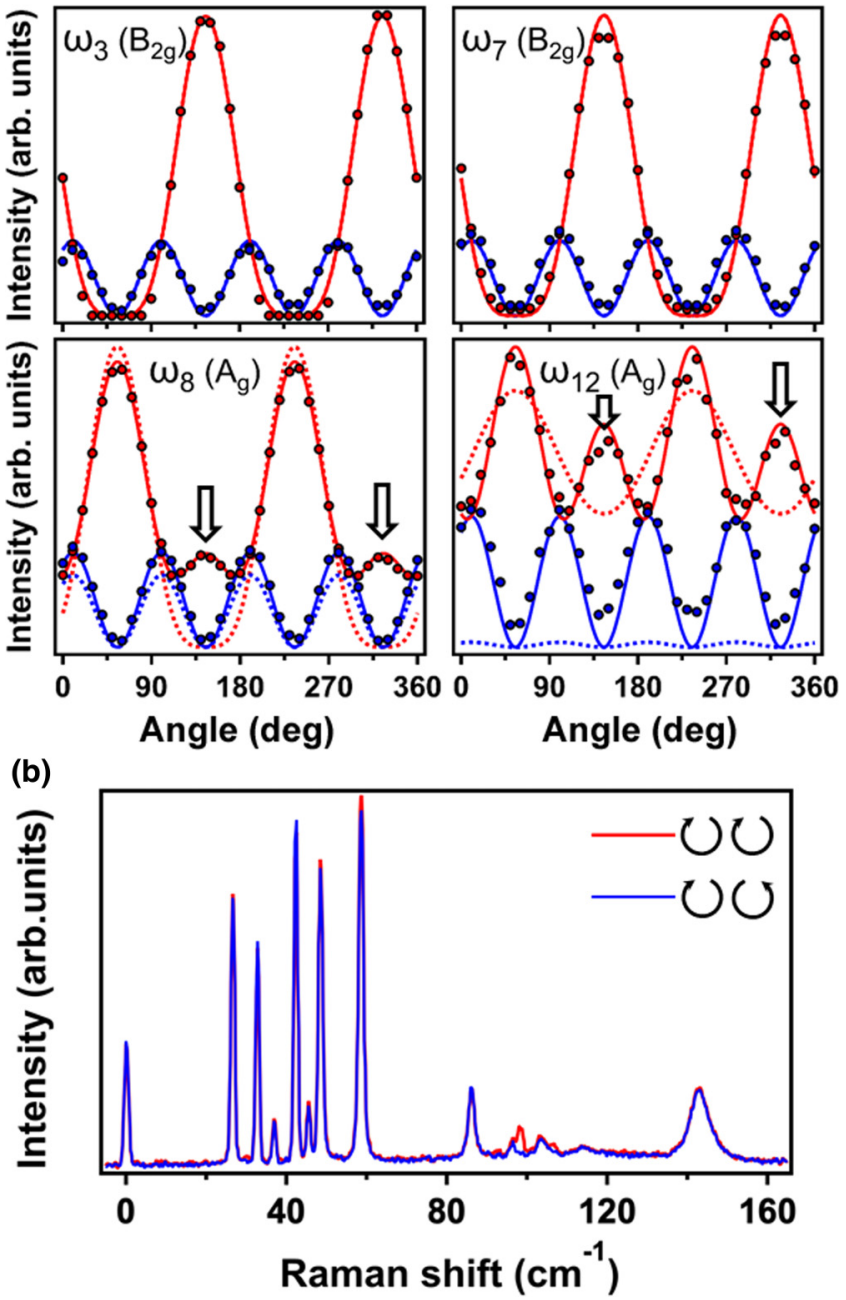

FIG. 3. (a) Fitting of PO data (solid circles) using Eq. (2) (dashed) and Eq. (4) (solid). The improved fit from Eq. (4) confirms the presence of strong birefringence. Red and blue curves represent parallel and cross configurations, respectively. The secondary maximum for $\mathrm{A}_{g}$ modes is shown by the arrows. (b) Circular polarization Raman spectra of MAPI in parallel (co-rotating) and cross (counterrotating) configurations.

propagation. Since the Raman measurements were done on a single crystallographic plane, $\left|\phi_{z}\right|$ cannot be determined by this specific model and cancels out during the calculation (see SM [34]). An additional phase factor $\delta$ is added to the angle $\theta$ to accommodate the birefringence effect so that $(\theta+\delta)$ is the actual angle of light polarization with respect to the crystal's axis. Since the wavelength of scattered light varies negligibly compared to the incident as regards birefringence, $\phi_{y}$ and $\delta$ were held fixed across all modes.

As shown in Fig. 3(a), Eq. (4) reproduces the intensity angular dependence of all the modes. Furthermore, we calculated the reversal coefficients [Eq. (3)] using the obtained Raman tensor components as additional verification to the fit results and got an excellent agreement between the model and experimental results (see SM [34], Table S2), including $\varrho=0$ for the mode at $\approx 98 \mathrm{~cm}^{-1}$. Previous work by Osorio et al. [20] measured polarized Raman of MAPI on a single angle 
and analyzed the symmetry using the isotropically averaged Raman intensity. Consequently, their assignments slightly differ from ours.

\section{CONCLUSION}

In conclusion, we unambiguously assigned the symmetries and atomic motions of the Raman-active lattice modes in the orthorhombic phase of MAPI. We achieved that by using Raman measurements with polarized light and density functional perturbation theory.

This allowed us to determine the crystal orientation, which is difficult to obtain at cryogenic temperatures across phase transitions. We observed anomalous behavior of the $\mathrm{A}_{g}$ modes which is attributed to strong birefringence effects. Our work demonstrates that the combination of experimental polarization-dependent Raman with theoretical calculations is an accurate and accessible method to obtain information about the structural dynamics of crystals, which otherwise would be difficult to acquire.

\section{ACKNOWLEDGMENTS}

The authors would like to thank Dr. Tsachi Livneh (NRC) for fruitful discussions, Dr. Ishay Feldman (WIS) for performing x-ray diffraction measurements, and Dr. Lior Segev (WIS) for developing the Raman software. This work was primarily supported by the NSF-BSF program, under NSF Grant No. DMR-1719353 and BSF Grant No. 2016650. R.S. acknowledges FGS-WIS for financial support. O.Y. acknowledges funding from: ISF (1861/17), BSF (2016650), ERC (850041-ANHARMONIC), Benoziyo Endowment Fund, Ilse Katz Institute, Henry Chanoch Krenter Institute, Soref New Scientists Start up Fund, Carolito Stiftung, Abraham \& Sonia Rochlin Foundation. Z.D. and L.G. acknowledge support from the US NSF, under Grant No. DMR-1719353. J.Z. acknowledges support from a VIEST Fellowship at the University of Pennsylvania. A.M.R. acknowledges support from the Office of Naval Research under Grant No. N00014-17-1-2574. The authors acknowledge computational support from the HighPerformance Computing Modernization Office of the U.S. Department of Defense.
[1] T. M. Brenner, D. A. Egger, L. Kronik, G. Hodes, and D. Cahen, Nat. Rev. Mater. 1, 15007 (2016).

[2] M. Grätzel, Nat. Mater. 13, 838 (2014).

[3] S. D. Stranks and H. J. Snaith, Nat. Nanotechnol. 10, 391 (2015).

[4] C. C. Stoumpos, C. D. Malliakas, and M. G. Kanatzidis, Inorg. Chem. 52, 9019 (2013).

[5] C. Wehrenfennig, G. E. Eperon, M. B. Johnston, H. J. Snaith, and L. M. Herz, Adv. Mater. 26, 1584 (2014).

[6] F. Panzer, C. Li, T. Meier, A. Köhler, and S. Huettner, Adv. Energy Mater. 7, 1700286 (2017).

[7] Y. Guo, O. Yaffe, D. W. Paley, A. N. Beecher, T. D. Hull, G. Szpak, J. S. Owen, L. E. Brus, and M. A. Pimenta, Phys. Rev. Mater. 1, 042401 (2017).

[8] D. A. Egger, A. Bera, D. Cahen, G. Hodes, T. Kirchartz, L. Kronik, R. Lovrincic, A. M. Rappe, D. R. Reichman, and O. Yaffe, Adv. Mater. 30, 1 (2018).

[9] M. Mayers, L. Z. Tan, D. A. Egger, A. M. Rappe, and D. R. Reichman, Nano Lett. 18, 8041 (2018).

[10] L. D. Whalley, J. M. Skelton, J. M. Frost, and A. Walsh, Phys. Rev. B 94, 220301(R) (2016).

[11] T. Baikie, N. S. Barrow, Y. Fang, P. J. Keenan, P. R. Slater, R. O. Piltz, M. Gutmann, S. G. Mhaisalkar, and T. J. White, J. Mater. Chem. A 3, 9298 (2015).

[12] I. P. Swainson, C. Stock, S. F. Parker, L. Van Eijck, M. Russina, and J. W. Taylor, Phys. Rev. B 92, 100303(R) (2015).

[13] M. Pazoki, A. Hagfeldt and T. Edvinsson, Characterization Techniques for Perovskite Solar Cell Materials (Elsevier, Amsterdam, 2020), Chap. 2, pp. 23-47.

[14] M. Sendner, P. K. Nayak, D. A. Egger, S. Beck, C. Müller, B. Epding, W. Kowalsky, L. Kronik, H. J. Snaith, A. Pucci, and R. Lovrinčić, Mater. Horizons 3, 613 (2016).

[15] I. Anusca, S. Balčiūnas, P. Gemeiner, Š. Svirskas, M. Sanlialp, G. Lackner, C. Fettkenhauer, J. Belovickis, V. Samulionis, M. Ivanov, B. Dkhil, J. Banys, V. V. Shvartsman, and D. C. Lupascu, Adv. Energy Mater. 7, 1700600 (2017).
[16] A. M. Leguy, A. R. Goñi, J. M. Frost, J. Skelton, F. Brivio, X. Rodríguez-Martínez, O. J. Weber, A. Pallipurath, M. I. Alonso, M. Campoy-Quiles, M. T. Weller, J. Nelson, A. Walsh, and P. R. Barnes, Phys. Chem. Chem. Phys. 18, 27051 (2016).

[17] D. L. Rousseau, M. Hill, R. P. Bauman, and S. P. S. Porto, J. Raman Spectrosc. 10, 253 (1981).

[18] W. Hayes and R. Loudon, Scattering of Light by Crystals (Dover Publications, New York, 2004), p. 103.

[19] O. Yaffe, Y. Guo, L. Z. Tan, D. A. Egger, T. Hull, C. C. Stoumpos, F. Zheng, T. F. Heinz, L. Kronik, M. G. Kanatzidis, J. S. Owen, A. M. Rappe, M. A. Pimenta, and L. E. Brus, Phys. Rev. Lett. 118, 136001 (2017).

[20] M. A. Pérez-Osorio, Q. Lin, R. T. Phillips, R. L. Milot, L. M Herz, M. B. Johnston, and F. Giustino, J. Phys. Chem. C $\mathbf{1 2 2}$ 21703 (2018)

[21] K. Nakada, Y. Matsumoto, Y. Shimoi, K. Yamada, and Y. Furukawa, Molecules 24, 626 (2019).

[22] F. Brivio, J. M. Frost, J. M. Skelton, A. J. Jackson, O. J. Weber, M. T. Weller, A. R. Goñi, A. M. Leguy, P. R. Barnes, and A. Walsh, Phys. Rev. B 92, 144308 (2015).

[23] C. Quarti, G. Grancini, E. Mosconi, P. Bruno, J. M. Ball, M. M. Lee, H. J. Snaith, A. Petrozza, and F. D. Angelis, J. Phys. Chem. Lett. 5, 279 (2014).

[24] B.-w. Park, S. M. Jain, X. Zhang, A. Hagfeldt, G. Boschloo, and T. Edvinsson, ACS Nano 9, 2088 (2015).

[25] P. Pistor, A. Ruiz, A. Cabot, and V. Izquierdo-Roca, Sci. Rep. 6, 35973 (2016).

[26] S. Ruan, M.-A. Surmiak, Y. Ruan, D. P. McMeekin, H. Ebendorff-Heidepriem, Y.-B. Cheng, J. Lu, and C. R. McNeill, J. Mater. Chem. C 7, 9326 (2019).

[27] M. Ledinský, P. Löper, B. Niesen, J. Holovský, S.-J. Moon, J.H. Yum, S. De Wolf, A. Fejfar, and C. Ballif, J. Phys. Chem. Lett. 6, 401 (2015).

[28] A. Poglitsch and D. Weber, J. Chem. Phys. 87, 6373 (1987). 
[29] P. S. Whitfield, N. Herron, W. E. Guise, K. Page, Y. Q. Cheng, I. Milas, and M. K. Crawford, Sci. Rep. 6, 35685 (2016).

[30] E. Kroumova, M. Aroyo, J. Perez-Mato, A. Kirov, C. Capillas, S. Ivantchev, and H. Wondratschek, Phase Transitions 76, 155 (2003).

[31] G. Schuck, D. M. Többens, M. Koch-Müller, I. Efthimiopoulos, and S. Schorr, J. Phys. Chem. C 122, 5227 (2018).

[32] E. B. Wilson, J. C. Decius, and P. C. Cross, Molecular Vibrations: The Theory of Infrared and Raman Vibrational Spectra (Dover Publications Inc, New York, 1980), p. 388.

[33] C. Kranert, C. Sturm, R. Schmidt-Grund, and M. Grundmann, Phys. Rev. Lett. 116, 127401 (2016).

[34] See Supplemental Material at http://link.aps.org/supplemental/ 10.1103/PhysRevMaterials.4.051601 for supporting experimental and computational details.

[35] J. Nestro and T. G. Spiro, J. Raman Spectrosc. 1, 539 (1973).
[36] Y. Wang, F. Yang, X. Li, F. Ru, P. Liu, L. Wang, W. Ji, J. Xia, and X. Meng, Adv. Funct. Mater. 29, 1904913 (2019).

[37] M. Wang and S. Lin, Adv. Funct. Mater. 26, 5297 (2016).

[38] N. Cho, F. Li, B. Turedi, L. Sinatra, S. P. Sarmah, M. R. Parida, M. I. Saidaminov, B. Murali, V. M. Burlakov, A. Goriely, O. F. Mohammed, T. Wu, and O. M. Bakr, Nat. Commun. 7, 13407 (2016).

[39] A. M. A. Leguy, P. Azarhoosh, M. I. Alonso, M. CampoyQuiles, O. J. Weber, J. Yao, D. Bryant, M. T. Weller, J. Nelson, A. Walsh, M. van Schilfgaarde, and P. R. F. Barnes, Nanoscale 8, 6317 (2016).

[40] W. Zheng, J. Yan, F. Li, and F. Huang, Photonics Res. 6, 709 (2018).

[41] S. Zhang, N. Mao, N. Zhang, J. Wu, L. Tong, and J. Zhang, ACS Nano 11, 10366 (2017).

[42] R. C. Jones, J. Opt. Soc. Am. 31, 488 (1941). 